\title{
Optimal Selection of Whey Processing Facilities and Technology
}

\author{
$\underline{\text { R. García-Flores }}^{a}$ and Pablo Juliano ${ }^{b}$

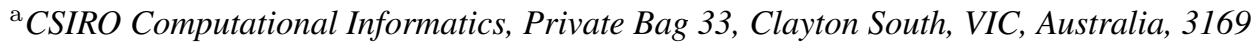 \\ ${ }^{\mathrm{b}}$ CSIRO Animal, Food and Health Sciences, Private Bag 16, Werribee, VIC, Australia, 3030 \\ Email: Rodolfo.Garcia-Flores@csiro.au
}

\begin{abstract}
A large proportion of cheese makers in many countries is made up of small enterprises that use less than 25,000 litres of milk per day. Currently, whey, a by-product of cheese making, goes mostly to waste as a highly contaminating effluent or to animal feed because small manufacturers do not have the capacity to preserve it to consistent quality levels. The optimisation model we present aims at achieving better whey utilisation by selecting a processing site from a cluster of cheese makers, suggesting transportation routes, and selecting among a number of technological alternatives for developing products from underutilised whey. The backbone of the model is a network design/facility location problem, with additional constraints that enable optimal technology selection for whey processing at the selected sites. We present preliminary results for a cluster of Victorian cheese makers to prove the feasibility of the approach. The model will economically assess the feasibility of developing distribution chains of underutilised whey in selected country regions.
\end{abstract}

Keywords: Facility Location, Network design, Dairy supply chain, Technology selection, Mixed-integer linear programming 


\section{INTRODUCTION}

Achieving better utilisation of the whey produced by small cheese manufacturers is a common challenge in countries where dairy is an important economic activity. In Australia, for example, the value of total dairy production amounts to US \$3.1 billion, of which US \$2.2 billion are exports [Klerkx and Nettle, 2013]. In this country, only half of the whey produced is converted into saleable products, while the remainder is disposed of, risking environmental problems rather than maximising returns [Durham et al., 2004]. Other countries face similar challenges: Wissmann et al. [2012] made an environmental and economic analysis about the problems caused by disposing of whey in the environment in Brazil. They noted that 50 thousand litres of whey thrown to the environment compare to the effluents of a settlement of 25 thousand inhabitants, and concluded that whey treatment can significantly reduce the cost of treating residuals, from $5.59 \%$ of the total operational costs to $3.98 \%$, which represents a relative reduction of $29 \%$.

Much unprocessed whey goes to waste or to animal feed because small manufacturers do not have the capacity to preserve it to consistent quality levels. To help these manufacturers add value, we should pay attention to a number of aspects of the problem. First, clusters of producers are often located in the same geographical area, so it makes sense in many cases to select a hub as a central processing facility. This facility will have a choice of products to market and technologies to obtain them, a summary of which is shown in Figure 1. Finally, the logistics (that is, the costs of transportation and the state of the road network connecting cheese producers) will also be an important fraction of the total cost of operating the whey supply chain.
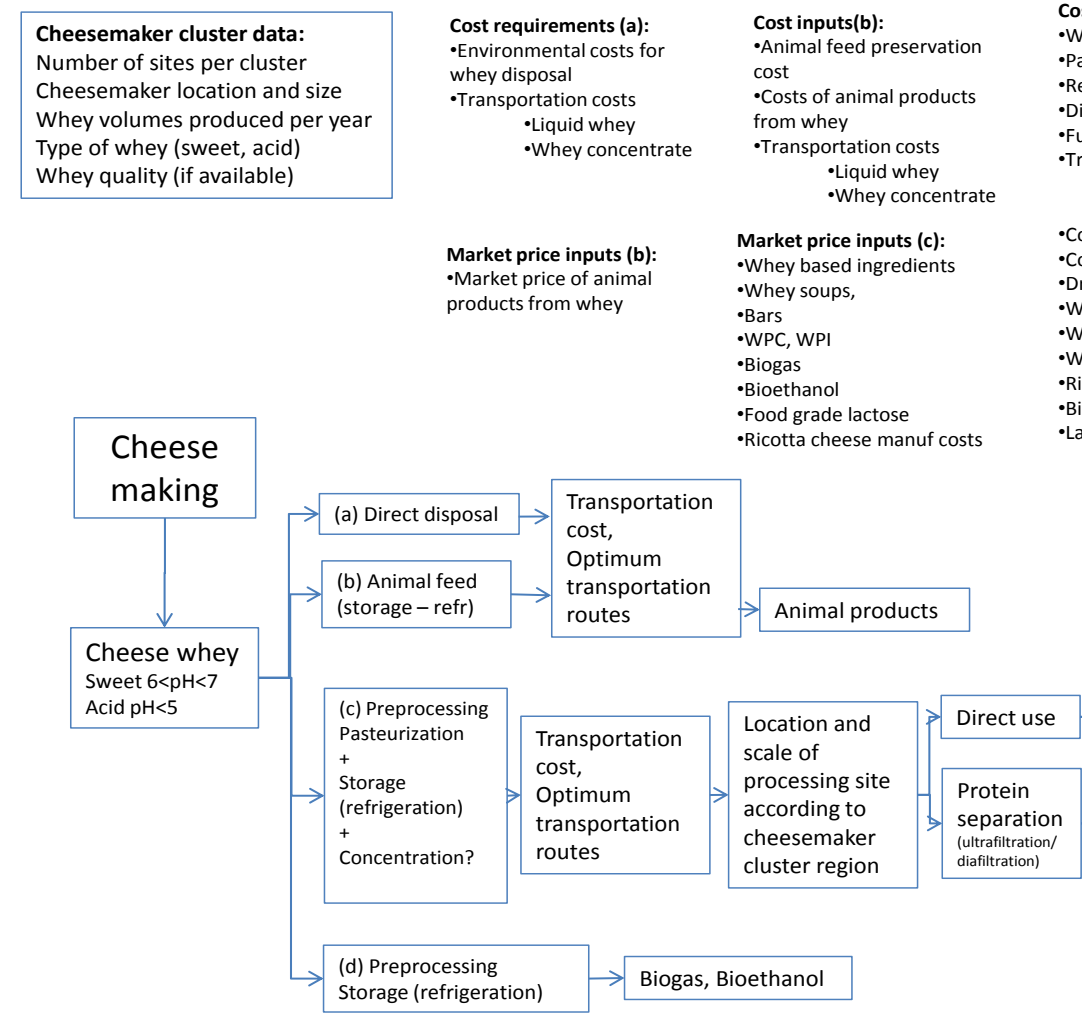

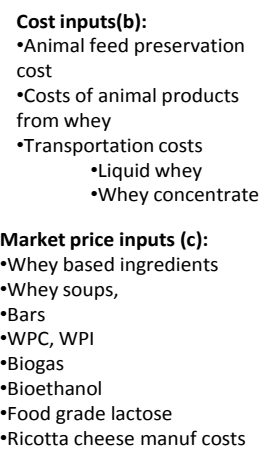

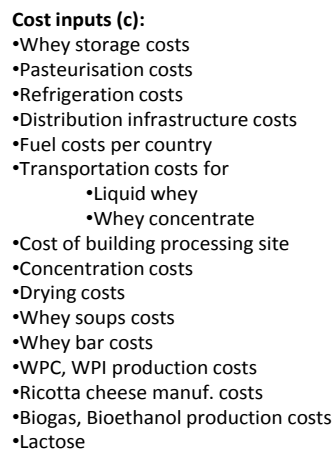

Figure 1. Diagram showing possible processing paths of whey.

The model that we introduce in this paper is a first step towards better understanding and improving the efficiency of the operations and logistics of whey production. It addresses the following questions: what is the optimal location of a processing facility and the most economically effective whey drying technology to install in this facility? What is the best design of the transportation network to take the whey to the selected processing facility? Previous research has addressed some aspects of this problem. Regarding the optimisation of the technology used for whey processing, Pinto and Giordano [2009] presented an integrated environment for simulation, monitoring, control and optimisation of a cheese whey refinery. This model was also developed 
partly to avoid the disposal of whey by small producers, and its emphasis was on validating experimental data. Regarding the logistics of production, Doganis and Sarimveis [2008] present a customized Mixed Integer Linear Programming (MILP) model for optimizing packaging lines of dairy products that consist of multiple parallel machines. Processing of raw materials is affected, among other factors, by protein composition. The model is used to optimise production of Greek yogurt producers. The interested reader may refer to Kelly [2007] and Jelen [2009] for details on whey properties, products and processing, which are beyond the scope of this paper.

This paper presents a model whose purpose is to select of a site as a whey processing facility among a set of candidates within a cluster of cheese manufacturers, and to select a technology combination for processing the whey at the selected site. It is structured as follows: Section 2 introduces the mathematical formulation of the model. Section 3 presents a case study using a cluster of small cheese producers from the state of Victoria and presents preliminary results. Finally, Section 4 discusses the implications of these results for the cheese makers.

\section{MATHEMATICAL MODEL}

The problem can be described as follows: a number of cheese producers located in a geographical cluster produce different amounts of whey. The whey produced by each cheese producer is transported to a processing facility whose location must be determined, but which will be selected from among the cheese producers in the cluster. The selected facility dries up the whey in one stage; the model does not distinguish whey by type, since the protein contents is the same for sweet and acid whey. Each of the candidate whey processing facilities can choose between two types of drier, or technologies, named D1 and D2. The driers differ in their efficiency and in their operating costs 1

The solution of the optimisation problem will indicate the transportation paths and the combination of whey processing facilities and technologies that maximise the profit of the cluster as a whole. It extends the network design and facility selection problem of Melkote and Daskin [2001b, a] to also allow technology selection.

\subsection{Network design and facility location}

We define a commodity $k$ which represents the amount of whey produced by a cheese maker and sent to the processing facility; $k$ does not represent a different type of whey. Let $x_{i j}$ be variables that indicate if link between nodes $i$ and $j$ are open between cheese makers and processing facility, $y_{i j}^{k}$ the fraction of demand flows of commodity coming from node $k$ on link $(i, j)$ between cheese makers and a facility, $z_{i}$ a variable that indicates if an whey processing facility is located at node $i$, and $w_{i}^{k}$ the fraction of demand of $k$ served by node $i$.

The details of the derivation of this part of the formulation, which assumes that the demand of the nodes selected as facility is in fact served by a super-node, can be found in Melkote and Daskin [2001b, a] and are not included here due to space limitations. The flow conservation constraint for the nodes selected as facilities $i$ is

$$
z_{i}+\sum_{j \in \mathcal{N}} x_{i j}=1 \quad \forall i \in \mathcal{N}
$$

which says that nodes selected as facilities fulfill the total processing demand, and that there are no outbound links transporting whey from the sites chosen as facilities. Here, $\mathcal{N}$ represents the number of sites. For the case where $i$ is the destination of commodity $k$, we have

$$
z_{k}+\sum_{i \in \mathcal{N}, i \neq k} w_{i}^{k}=1 \quad \forall k \in \mathcal{N}
$$

which says that the demand of all other nodes that are not facilities is supplied by the facilities. Equations (1) and (2) specify zero demand for the nodes that are not selected as facilities. Conservation constraints for

\footnotetext{
1 Jelen 2009] mentions that whey is dried using either the spray drying technique, which is more expensive and does not damage the protein, or the cheaper roller drying technique, which is detrimental to the quality of the product. D1 and D2 may correspond to these technologies.
} 
R. García-Flores and P. Juliano, Optimal Selection of Whey Processing Facilities

selected and non-selected links in the network design are

$$
\begin{aligned}
x_{k i}+\sum_{j \in \mathcal{N}: j \neq k} y_{j i}^{k} & =\sum_{j \in \mathcal{N}} y_{i j}^{k}+w_{i}^{k} \quad \forall i, k \in \mathcal{N}, i \neq k, \forall(k, i) \in \mathcal{L}, \\
\sum_{j \in \mathcal{N}} y_{j i}^{k} & =\sum_{j \in \mathcal{N}} y_{i j}^{k}+w_{i}^{k} \quad \forall i, k \in \mathcal{N}, i \neq k, \forall(k, i) \notin \mathcal{L},
\end{aligned}
$$

where $w_{i}^{k}$ is the fraction of demand of $k$ served by node $i$ and $\mathcal{L}$ is the set of road segments. Constraints (3) and (4) are the conservation equations for links that remain open in the final network design and those that do not, respectively. Also, flow is only permitted in the links that are part of the transportation design from cheese makers to facilities,

$$
y_{i j}^{k} \leq x_{i j} \quad \forall(i, j) \in \mathcal{L}, \forall k \in \mathcal{N}, i \neq k,
$$

and demand from an individual cheese maker is served by a node only if this node is selected as a facility,

$$
w_{i}^{k} \leq z_{i} \quad \forall i, k \in \mathcal{N}, i \neq k .
$$

We only care if links are open, regardless of their direction.

$$
x_{i j}+x_{j i} \leq 1 \quad \forall(i, j) \in \mathcal{L} .
$$

To assess the trade-off between allocating resources to operations or facilities, we could either add the budget constraint

$$
\sum_{i \in N} f_{i} z_{i}+\sum_{(i, j) \in L} c_{i j} x_{i j} \leq B
$$

where $f_{i}$ is the fixed cost of constructing a facility at $i, B$ is the budget allocated to facility construction and $c_{i j}$ is the cost of constructing link $(i, j)$ and minimise transportation costs. Alternatively, we can add the terms on the left hand side of $(8)$ to the objective function and do without this constraint.

Because this is a concave cost flow problem, as pointed out by Ahuja et al. [1993], the optimal solution of this problem is a directed, rooted spanning tree with the super-node as the root node. By definition, all nodes of a directed, rooted spanning tree except the root node have exactly one outbound link (or out degree) of one. The following constraints are a consequence of this property:

$$
\begin{gathered}
y_{i j}^{i}=x_{i j} \quad \forall(i, j) \in \mathcal{L}, \\
w_{i}^{i}=z_{i} \quad \forall i \in \mathcal{N} .
\end{gathered}
$$

\subsection{Technology selection}

Technologies D1 and D2 differ in their efficiencies, $\eta_{1}$ and $\eta_{2}$, respectively. Let $\delta_{i e}$ be indicator variables that take the value one if site $i$ selects technology $e$ and zero otherwise, and

$$
\sum_{i \in \mathcal{N}} \sum_{e \in \mathcal{E}} \delta_{i e}=1
$$

so that only one combination of site and technology is chosen. Define the mass fraction of protein in whey as $\phi$. Because only one site is selected as processing facility, $Q=\sum_{k \in \mathcal{N}} d_{k}$ is the total amount of whey processed. The total amount of protein to be produced is

$$
Q \phi \eta_{1} z_{i} \delta_{i 1}+Q \phi \eta_{2} z_{i} \delta_{i 2}
$$

If we consider that the cost of constructing a facility at node $i$ consists mostly of the cost of setting up the selected technology $g_{i e}$, the facility setup cost (i.e., the terms $f_{i} z_{i}$ in constraint (8)) becomes

$$
g_{i 1} z_{i} \delta_{i 1}+g_{i 2} z_{i} \delta_{i 2},
$$

Multiplying expression (12) by the selling price of whey protein $p$ produces the income from sales of finished product. These expressions are non-linear, but because they contains products of binary variables, they can 
R. García-Flores and P. Juliano, Optimal Selection of Whey Processing Facilities

be linearised by adding additional binary variables as follows: let $\pi_{i e}$ replace the product $z_{i} \delta_{i e}$ so that (12) becomes

$$
Q \phi \eta_{1} \pi_{i 1}+Q \phi \eta_{2} \pi_{i 2}
$$

the same applies to (13). With these new variables, constraint (8) can be written as

$$
\sum_{i \in \mathcal{N}} \sum_{e \in \mathcal{E}} g_{i e} \pi_{i e}+\sum_{(i, j) \in L} c_{i j} x_{i j} \leq B
$$

To guarantee that every $\pi_{i e}$ becomes zero if either of the binary variables in the product term is zero, we introduce the following constraints $(\mathcal{E}$ is the set of technologies):

$$
\pi_{i e} \leq z_{i} \quad \forall i \in \mathcal{N}, \forall e \in \mathcal{E}, \quad \text { and } \quad \pi_{i e} \leq \delta_{i e} \quad \forall i \in \mathcal{N}, \forall e \in \mathcal{E},
$$

To ensure that $\pi_{i e}$ equals one if $z_{i}$ and $\delta_{i e}$ equal one, we introduce

$$
z_{i}+\delta_{i e}-\pi_{i e} \leq 1 \quad \forall i \in \mathcal{N}, \forall e \in \mathcal{E}
$$

The model is constrained to selecting one facility, $\sum_{i \in \mathcal{N}} z_{i}=1$, which has the advantage of ensuring that the total amount of whey produced $Q$ and which ends up in a single place is known before the solution is calculated. We also add integrality and nonnegativity constraints.

\subsection{Objective function}

The objective function maximises the profit of the cluster of cheese makers, expressed as the income from the whey protein sales, minus the cost of setting up the whey processing facility, minus the variable transportation costs, minus fixed transportation costs. Assuming that the number of facilities is constrained to one, this is

$$
\begin{aligned}
\text { Maximise } & p Q \phi \sum_{e \in \mathcal{E}} \eta_{e} \sum_{i \in \mathcal{N}} \pi_{i e}-\sum_{e \in \mathcal{E}} \sum_{i \in \mathcal{N}} g_{i e} \pi_{i e}-\sum_{(i, j) \in \mathcal{L}} \sum_{k \in \mathcal{N}, k \neq i} T C_{i j} y_{i j}^{k}-\sum_{(i, j) \in L} c_{i j} x_{i j} \\
= & \sum_{e \in \mathcal{E}} \sum_{i \in \mathcal{N}}\left(p Q \phi \eta_{e}-g_{i e}\right) \pi_{i e}-\sum_{(i, j) \in \mathcal{L}} \sum_{k \in \mathcal{N}, k \neq i} T C_{i j} y_{i j}^{k}-\sum_{(i, j) \in L} c_{i j} x_{i j}
\end{aligned}
$$

where $T C_{i j}$ are the transportation costs in link $(i, j) \in \mathcal{L}, i, j \in \mathcal{N}$.

\section{CASE STUDY AND RESULTS}

To illustrate the methodology, a set of 23 hypothetical cheese makers from the region of Loddon Mallee in Victoria (Figure 2) was selected and assigned random setup costs for technology a as $\mathrm{N}(\mu=\$ 60000.00, \sigma=$ $\$ 10000)$ for technology D1, and $\mathrm{N}(\mu=\$ 50000.00, \sigma=\$ 10000)$ for technology D2. The efficiencies of the technologies are assumed to be $\epsilon_{1}=0.95$ and $\epsilon_{2}=0.75$ for D1 and D2, respectively. The selling price is set to $\$ 800.00$ per tonne of whey, and the assumed transportation cost is $\$ 40.00$ per tonne of whey. Production is assigned randomly according to $\mathrm{N}(\mu=90$ ton, $\sigma=20)$. The links in the Figure follow approximately the existing road network. All calculations were made using CPLEX 12.52 in a 64-bit Intel Xeon CPU with one processor of eight cores $(2.27 \mathrm{GHz})$ each and 16 GB of RAM. The problem has 2084 variables and 1934 constraints and was coded in Clojure ${ }^{3}$ with an Excel interface. Figure 3 shows the resulting transportation network, with Wyuna and D1 selected as the processing facility and drying technology, respectively. The selected facility is central, as it would be expected when the production from all sites is not too different, and the optimal configuration yields a net profit of $\$ 7066.45$, of which $\$ 156028.00$ is sales income, $\$ 90587.55$ are total transportation costs, and $\$ 58374.00$ are site setup costs. It is important to note at this stage that the values are all assumed and the present example is introduced simply to demonstrate the validity of the approach.

\section{CONCLUDING REMARKS}

Achieving better use of the whey produced in cheese making is an economically and environmentally attractive proposition. In this paper, we have introduced a model that determines the optimal location of a processing

2 http://www-01.ibm.com/software/integration/optimization/cplex-optimizer/ accessed on the 8 of April 2013.

3 http://clojure.org/ 
R. García-Flores and P. Juliano, Optimal Selection of Whey Processing Facilities

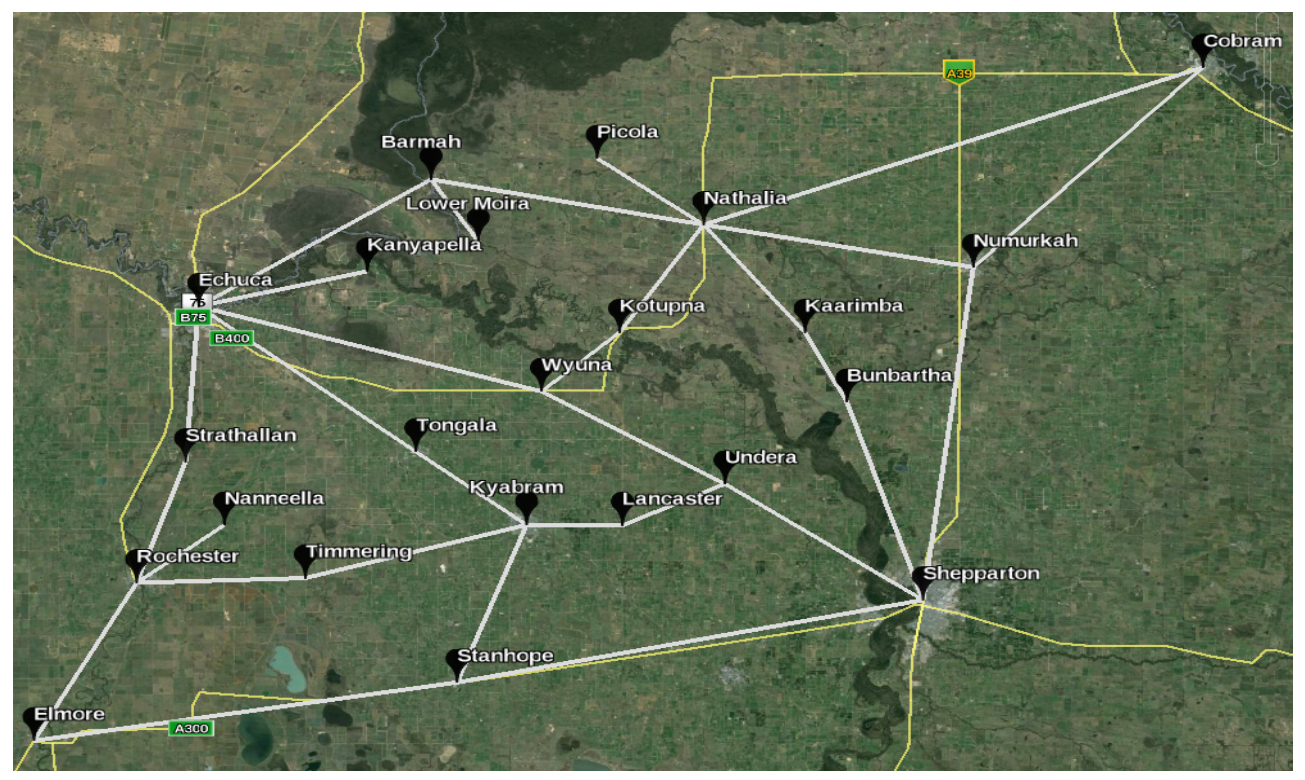

Figure 2. Map showing hypothetical cheese makers and connecting road network.

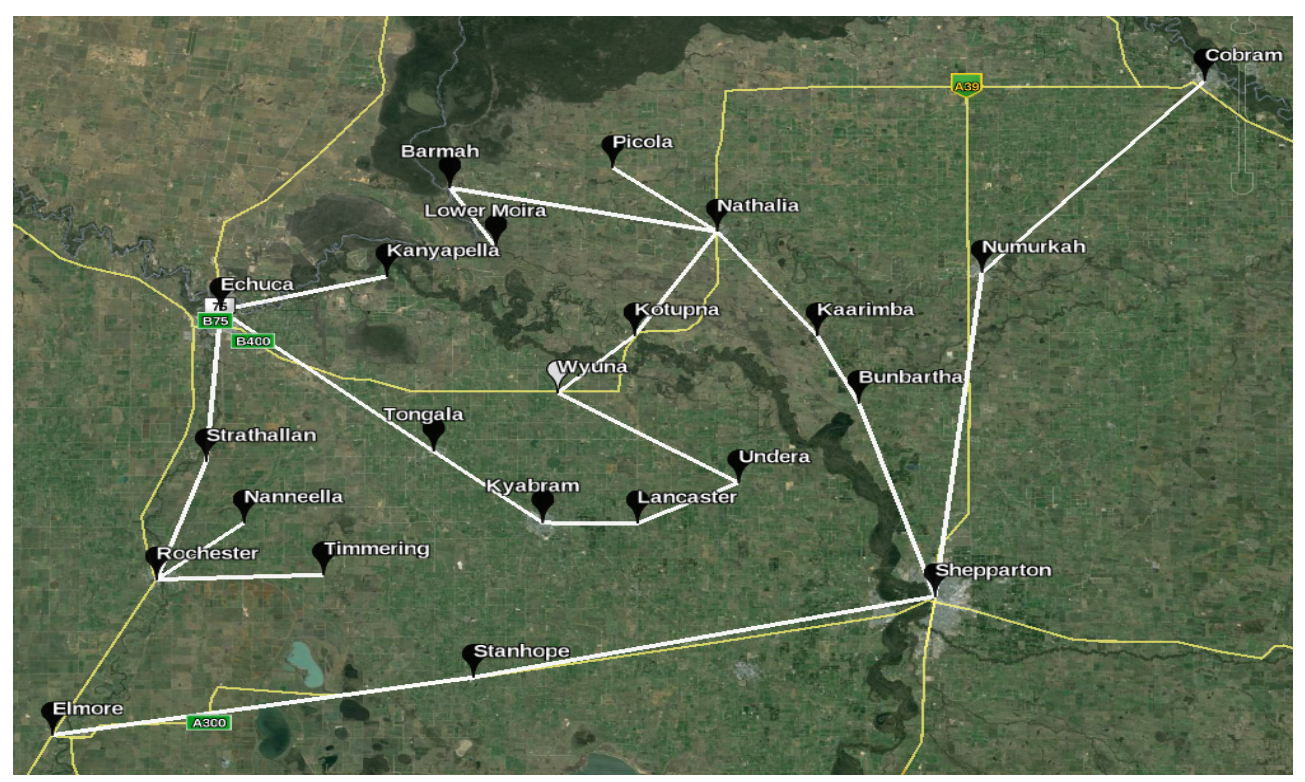

Figure 3. Map showing the optimal whey transportation network for the region under study. The technology to set up in Wyuna is D1. 
facility, the most economically effective whey drying technology to install in this facility, and the best design of the transportation network to take the whey to the selected processing facility.

The optimal selection of routes, processes and facilities from among all the available options is not easy even for small supply chains, due to the combinatorial explosion. We have demonstrated the feasibility of the approach by using a hypothetical cluster of cheese producers in the Loddon Mallee region of Victoria.

A more realistic optimisation model of an actual cluster of manufacturers must consider as parameters the number of producers, their location and size, volume of whey produced per site, and types and qualities of whey powder. The model should also also consider processing costs, such as transportation, sales and technology set-up, based on actual economic conditions.

The model presented here is limited to selecting only one facility and one of only two possible technologies. Regarding the single facility, this limitation can be easily overcome by defining the amount of whey processed in each selected facility as $q_{i}=\sum_{k \in \mathcal{N}} w_{i}^{k} d_{k}$, at the expense of a quick increase in additional binary variables needed to represent the products of binary variables. Expanding the model to incorporate more of the technologies listed in Figure 1 may require significant changes.

\section{REFERENCES}

Ahuja, R., T. Magnanti, and J. Orlin (1993). Network Flows. Englewood Cliffs, NJ: Prentice Hall.

Doganis, P. and H. Sarimveis (2008, March). Optimal production scheduling for the dairy industry. Annals of Operations Research 159(1), 315-331.

Durham, R., R. Sleigh, and J. Hourigan (2004, August). Pharmaceutical lactose: a new whey with no waste. Australian Journal of Dairy Technology 59(2), 138-141.

Jelen, P. (2009). Dried Whey, Whey Proteins, Lactose and Lactose Derivative Products, Volume Dairy Powders and Concentrated Products (ed A. Y. Tamime), Chapter 7, pp. 255-267. Oxford, UK: Wiley-Blackwell.

Kelly, A. (2007). Cheese problems solved, Chapter 75, pp. 163-165. Number 147 in Woodhead Publishing Series in Food Science, Technology and Nutrition. Woodhead Publishing Ltd.

Klerkx, L. and R. Nettle (2013). Achievements and challenges of innovation co-production support initiatives in the australian and dutch dairy sectors: a comparative study. Food Policy (40), 74-89.

Melkote, S. and M. Daskin (2001a). Capacitated facility location/network design problems. European Journal of Operational Research 129, 481-495.

Melkote, S. and M. Daskin (2001b). An integrated model of facility location and transportation network design. Transportation Design Part A 35, 515-538.

Pinto, G. and R. Giordano (2009, August). Bioprocess systems engineering applied to the production of protein hydrolysates in a multipurpose plant. In Proceedings of the 10th International Symposium on Process Systems Engineering, Volume 27 of Computer-aided Chemical Engineering, pp. 1887-1892. Elsevier Science BV.

Wissmann, M., A. Hein, J. Follmann, and N. Rachow (2012). Environmental costs: analysis of its impact and importance in the pursuit of eco-efficiency in an industry of cheese. Custos e Agronegocio 8(3), 2-23. 